\title{
Clinical Study \\ Low Vital Capacity and Electrocardiographic ST-T Abnormalities in Asymptomatic Adults
}

\author{
Kei Nakajima, ${ }^{1,2}$ Yulan Li, ${ }^{1}$ Hiroshi Fuchigami, ${ }^{3}$ and Hiromi Munakata ${ }^{2}$ \\ ${ }^{1}$ Division of Clinical Nutrition, Department of Medical Dietetics, Faculty of Pharmaceutical Sciences, Josai University, \\ 1-1 Keyakidai, Sakado, Saitama 350-0295, Japan \\ ${ }^{2}$ Department of Internal Medicine, Social Insurance Omiya General Hospital, 453 Bonsai, Kita, Saitama 331-0805, Japan \\ ${ }^{3}$ Department of Health Care Center, Social Insurance Omiya General Hospital, 453 Bonsai, Kita, Saitama 331-0805, Japan \\ Correspondence should be addressed to Kei Nakajima, keinaka@josai.ac.jp
}

Received 18 February 2012; Accepted 24 March 2012

Academic Editor: Hisako Matsumoto

Copyright ( $) 2012$ Kei Nakajima et al. This is an open access article distributed under the Creative Commons Attribution License, which permits unrestricted use, distribution, and reproduction in any medium, provided the original work is properly cited.

Studies have shown that low forced vital capacity (LFVC) is associated with atherosclerosis. However, it is unclear whether LFVC is associated with resting electrocardiographic ST-T abnormalities, a common finding that is prognostic for cardiovascular events. Therefore, pulmonary functions, ST-T abnormalities defined with Minnesota Code, and cardiometabolic risk factors were examined in a cross-sectional study of 1,653 asymptomatic adults without past history of coronary heart diseases. The prevalence of diabetes, metabolic syndrome, and ST-T abnormalities significantly increased with decreasing percent of predicted forced vital capacity (\%PFVC). ST-T abnormalities were observed in 73 subjects (4.4\% in total). Multiple logistic regression analysis showed that, compared with the highest quartile of $\%$ PFVC $(\geq 99.7 \%)$, the lowest quartile of $\%$ PFVC $(\leq 84.2 \%)$ was persistently associated with ST-T abnormalities even after further adjustment for diabetes or metabolic syndrome (odds ratio (95\%CI): 2.44 (1.165.14 ) and 2.42 (1.15-5.10), resp.). Similar trends were observed when subjects were divided into quartiles according to percent of predicted forced expiratory volume in 1 second $\left(\mathrm{FEV}_{1}\right)$, but not the ratio of $\mathrm{FEV}_{1} / \mathrm{FVC}$. In conclusion, LFVC may be associated with ST-T abnormalities independent of metabolic abnormalities in asymptomatic adults, suggesting a plausible link between impaired pulmonary defects and cardiovascular diseases.

\section{Introduction}

Accumulating evidence has shown that not only impaired obstructive pulmonary function but also low forced vital capacity (LFVC) and impaired restrictive pulmonary function are associated with increased risk of mortality, partially owing to cardiovascular disease $[1,2]$. Some studies have shown that LFVC is associated with arterial stiffness assessed by pulse-wave velocity $[3,4]$ and coronary artery calcification [5], which may reflect systemic atherosclerosis. In addition, LFVC and a restrictive pattern have been associated with critical metabolic abnormalities such as type 2 diabetes and metabolic syndrome (MetS) $[2,5-8]$, which are often followed by the development of cardiovascular disease $[9$, 10]. To date, however, the association between and the underlying mechanism of such pulmonary function defects and subclinical cardiovascular disease have been poorly understood.
Meanwhile, ST depression, T-wave abnormalities, or both (ST-T abnormalities), particularly minor ST-T abnormalities, on the resting electrocardiogram are commonly observed in the general population without coronary heart disease [11-15]. Major ST-T abnormalities are an independent predictor for stroke [13], cardiovascular events, and increased mortality risk in people without heart disease at baseline $[11,12]$. Likewise, many studies have provided evidence that even minor ST-T abnormalities are associated with increased risk for future cardiovascular disease independent of traditional risk factors [14, 16, 17], although the prognostic significance of minor ST-T abnormalities has not been well established [14].

In the light of this evidence, we hypothesized that impaired pulmonary functions, especially LFVC, may be associated with ST-T abnormalities irrespective of cardiometabolic risk factors, diabetes, and MetS. So far, no large epidemiological studies have examined the associations 
between impaired lung functions and ST-T abnormalities in asymptomatic individuals without overt heart disease.

Therefore, we examined lung functions, ST-T abnormalities that comprised major and minor ST-T abnormalities, cardiometabolic risk factors, diabetes, and MetS in a crosssectional study of asymptomatic adults who underwent an annual checkup. Abnormal pulmonary function was evaluated by continuous pulmonary function variables including the percentage of predicted forced vital capacity (\%PFVC).

\section{Methods}

The current study represents a series of studies performed in collaboration with Josai University, Sakado, Japan, and Social Insurance Omiya General Hospital, Saitama, Japan, that were conducted to explore the causalities and mechanism in lifestyle-related diseases. The study design and protocol have been described elsewhere [8]. The protocol was approved by the Ethical Committee of Josai University and the Council of the Hospital, and informed consent was obtained from all participants.

\section{Subjects}

Asymptomatic healthy subjects $(n=2,488)$ were randomly recruited from those who underwent complete medical checkups at their own will at the Health Care Center attached to Omiya General Hospital between April 2009 and March 2010. Most lived or worked close to the hospital (a small portion was treated in the hospital). Individuals who required immediate treatment for suspected cancers, pneumothorax, or infectious pneumonia, including tuberculosis, were not included from the beginning. All recruited subjects, who were free from overt disability and hemiplegia, completed a questionnaire about their lifestyle characteristics. Exclusion criteria of subjects and a flow chart are shown in Figure 1. Since people less than 45 years old are less likely to have ST-T abnormalities, first, subjects outside the age limitation of 45 to 80 years were excluded. Second, subjects with a high C-reactive protein (CRP) level $(>10 \mathrm{mg} / \mathrm{L})$ were excluded because of latent critical diseases or inflammation. Third, subjects with abnormal electrocardiograms, with the exception of ST-T abnormalities, were excluded. Subjects with a self-reported medical history of overt coronary heart disease were also excluded. Finally, after additional exclusion of subjects with suspected interstitial pneumonia based on chest X-ray findings and those without available spirometry data, 1,653 subjects (1,014 men and 639 women) were eligible for this analysis.

\section{Laboratory Measurements and Determination of MetS and Diabetes}

Anthropometric measurements and laboratory tests were carried out after an overnight fast. Waist circumference was measured at the height of the navel. Clinical and biochemical variables were measured automatically with standard methods using an autoanalyzer (Hitachi, Tokyo, Japan). The diagnosis of MetS was based on the modified Third Report of the National Cholesterol Education Program Expert Panel/Adult Treatment Panel criteria [9] with the following cutoff values: (1) systolic blood pressure of $\geq 130 \mathrm{mmHg}$ and diastolic blood pressure of $\geq 85 \mathrm{mmHg}$; (2) triglycerides of $\geq 150 \mathrm{mg} / \mathrm{dL}$; (3) low high-density lipoprotein of $<40 \mathrm{mg} / \mathrm{dL}$ for men and $<50 \mathrm{mg} / \mathrm{dL}$ for women; (4) fasting plasma glucose of $\geq 100 \mathrm{mg} / \mathrm{dL}$; (5) waist circumference of $\geq 90 \mathrm{~cm}$ for men and $\geq 80 \mathrm{~cm}$ for women. We took ethnic-specific values for waist circumference into consideration. MetS was diagnosed in subjects fulfilling three or more of the above five criteria. Subjects receiving medication for any of these components were defined as having the component. Diabetes was defined as a fasting plasma glucose of $\geq 126 \mathrm{mg} / \mathrm{dL}$ or $\mathrm{HbAlc}$ of $\geq 6.5 \%$ according to the American Diabetes Association criteria [18], or treatment with oral hypoglycemic drugs or insulin. $\mathrm{HbAlc}$ was converted to National Glycohemoglobin Standardization Program (NGSP) levels by the formula HbAlc $(\%)($ NGSP $)=$ HbAlc $(J D S)(\%)+0.4 \%$ [19].

\section{Spirometry}

Pulmonary function tests were performed with a spirometry analyzer (Autospiro-507, Minato Medical Science Co., Ltd., Osaka, Japan). The test was performed by trained technicians with the subject in a standing position. The \%PFVC, percentage of predicted forced expiratory volume in 1 second $\left(\% \mathrm{PFEV}_{1}\right)$, and ratio of forced expiratory volume in 1 second to observed forced vital capacity $\left(\mathrm{FEV}_{1} / \mathrm{FVC}\right)$ were calculated as in our previous report [8]. We quoted the standard predicted values for $\mathrm{FVC}, \mathrm{FEV}_{1}$, and $\mathrm{FEV}_{1} / \mathrm{FVC}$ from data published by the Japanese Respiratory Society in 2001 [20].

\section{Electrocardiogram}

Standard 12-lead electrocardiograms were recorded with an electrocardiogram recorder (Cardio Base FDX-4521, Fukuda Denshi Co., Ltd., Tokyo, Japan). ST-T abnormalities were defined with the Minnesota Code [21]. Minor ST-T abnormalities included the following: ST junction depression of $<0.5 \mathrm{~mm}$ (MC 4-3); ST junction depression of $>1 \mathrm{~mm}$ and ST segment ascent, that is, upslope (MC 4-4); T wave flat, diphasic, or inverted by $<1 \mathrm{~mm}$ (MC 5-3). Major ST-T abnormalities included (MC 4-1), (MC 4-2), (MC 5-1), and (MC 5-2). The findings were first determined by experienced physicians and subsequently confirmed by trained medical laboratory technicians, all of whom exclusively belong to the Social Insurance Omiya General Hospital and were blinded to individuals' data. The minor and major ST-T abnormalities were combined and analyzed together in this study because, on the checkup sheets, minor and major ST abnormalities were recorded together indistinguishably.

\section{Statistical Analysis}

Data are expressed as means \pm SD or median/geometric mean (interquartile range). Subjects were divided into quartiles according to continuous pulmonary function variables: 


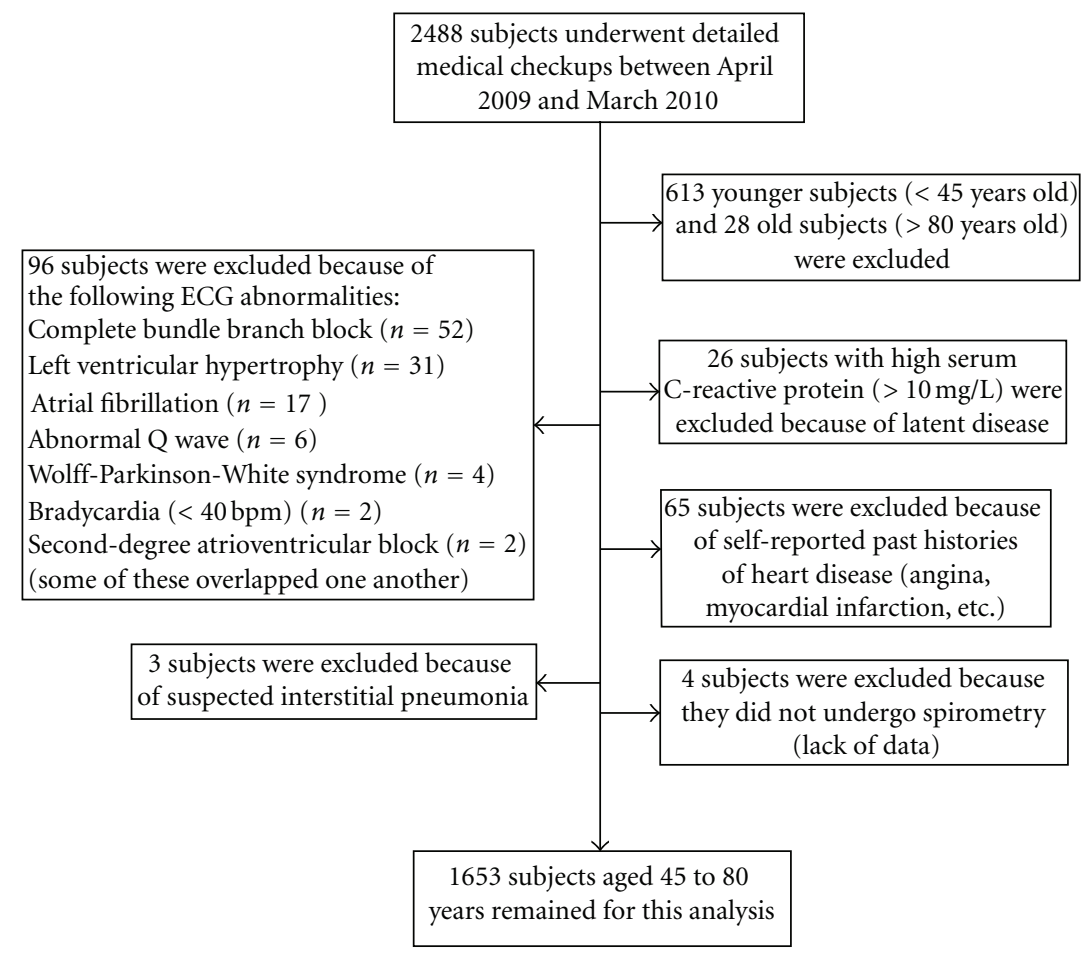

FIgURE 1: Exclusion criteria of subjects and flow chart.

\%PFVC, \% $\mathrm{PFEV}_{1}$, and $\mathrm{FEV}_{1} / \mathrm{FVC} . P$ values for continuous variables and categorical variables were determined with ANOVA and the $\chi^{2}$-test, respectively.

Multivariate logistic regression models were used to examine the associations between the lowest quartiles (Q1) of lung functions and ST-T abnormalities compared with the corresponding highest quartiles (Q4), controlling for clinical confounding factors including diabetes and MetS. This analysis yielded odds ratios (OR) and 95\% confidential intervals (95\% CI). Tests for linear trends ( $P$ for trend) were calculated by treating quartile categories (Q1-Q4) as a continuous variable (i.e., 1-4), and the same model analysis was conducted. In this study, hypertension $(\geq 130 / 85 \mathrm{mmHg})$ was considered as a special confounder that substantially interferes with the associations because hypertension is likely to affect ST-T abnormalities and the decline of FVC [13, 22]. Statistical analyses were performed using SPSS software version 18.0 (SPSS-IBM, Chicago, IL) and Statview version 5.0 (SAS Institute, Cary, NC). Values of $P<0.05$ were considered to be statistically significant.

\section{Results}

Overall, most subjects in this study had relatively good profiles in terms of anthropometric and biochemical parameters, including pulmonary functions tests (Table 1). The prevalence of cardiometabolic risk factors, diabetes, MetS, and ST-T abnormalities significantly increased with decreasing \%PFVC (toward Q4).

Multiple logistic analysis showed that compared with the highest quartile of \%PFVC ( $\geq 99.7 \%)$ (Q1), the lowest quartile ( $\leq 84.2 \%)$ (Q4) was significantly associated with ST-T abnormalities (Table 2). This association remained significant even after adjustment for age, sex, smoking, alcohol consumption, frequency of exercise, self-reported past history of stroke, waist circumference, and CRP (both as a continuous variable), with significant $P$ for trends. Moreover, extended adjustment for diabetes (Model 4) or MetS (Model 5) attenuated but did not remarkably alter the associations. In these conditions, diabetes and MetS were significantly associated with ST-T abnormalities (OR (95\% CI), 2.18 (1.18-4.03), $P=0.01$ and 2.20 (1.23-3.93), $P=$ 0.008 , resp.; data not shown).

Likewise, similar trends were observed when subjects were divided into quartiles according to $\% \mathrm{PFEV}_{1}$. However, observed associations between lowest quartile of $\% \mathrm{PFEV}_{1}$ and ST-T abnormalities were not significant after adjustment for diabetes or MetS. In contrast, no significant associations between lowest quartile of $\mathrm{FEV}_{1} / \mathrm{FVC}$ and ST-T abnormalities were observed in comparison with the highest quartile of $\mathrm{FEV}_{1} / \mathrm{FVC}$, irrespective of adjustments for confounders.

Meanwhile, the lowest quartile of \%PFVC was significantly associated with hypertension $(\geq 130 / 85 \mathrm{mmHg})$ (OR (95\%CI), 1.76 (1.30-2.38), $P=0.0003$, data not shown) compared with the highest quartile, even after full adjustments for confounders in Model 3 of Table 2. However, the significant association between LFVC and ST-T abnormalities persisted after further adjustments for confounders in Model 3 of Table 2, plus hypertension or medication for hypertension (OR (95\%CI), 2.43 (1.15-5.11), $P=0.02$ and $2.58(1.23-5.40), P=0.01$, resp., data not shown). 


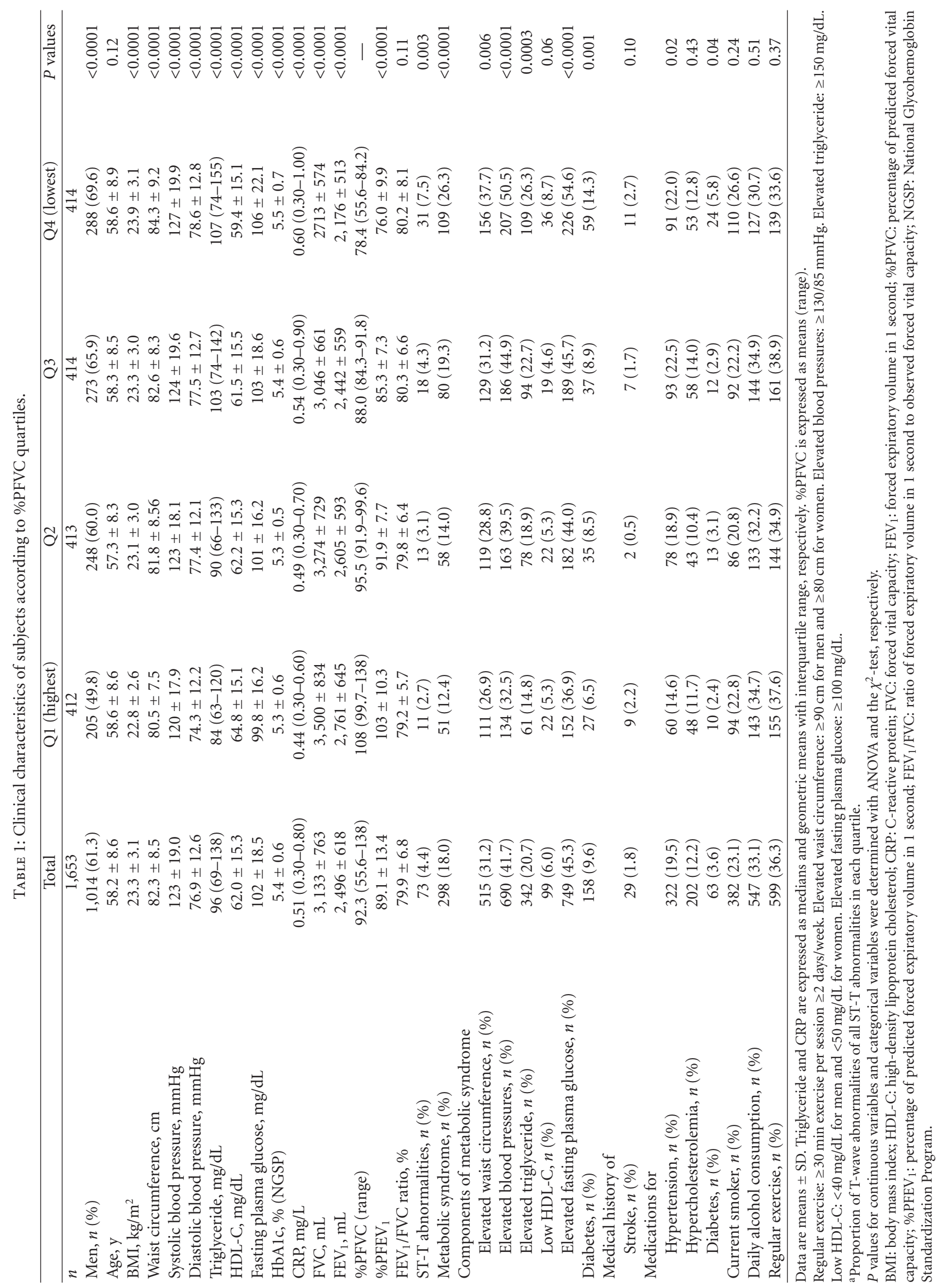


TABLE 2: Odds ratio (95\% CI) of lung function quartiles for ST-T abnormalities.

\begin{tabular}{|c|c|c|c|c|c|}
\hline & $\begin{array}{c}\text { Q1 } \\
\text { (highest) }\end{array}$ & Q2 & Q3 & Q4 (lowest) & $P$ for trend $*$ \\
\hline$\%$ PFVC, $n$ & 412 & 413 & 414 & 414 & \\
\hline Model 1 & 1 & $1.19(0.52-2.68)$ & $1.66(0.77-3.55)$ & $2.95(1.46-5.95)^{\dagger}$ & 0.0007 \\
\hline Model 2 & 1 & $1.33(0.59-3.03)$ & $1.83(0.85-3.95)$ & $3.27(1.60-6.69)^{\dagger}$ & 0.0004 \\
\hline Model 3 & 1 & $1.30(0.56-3.00)$ & $1.74(0.79-3.81)$ & $2.59(1.23-5.42)^{\S}$ & 0.006 \\
\hline Model 4 & 1 & $1.29(0.56-2.97)$ & $1.74(0.79-3.83)$ & $2.44(1.16-5.14)^{\S}$ & 0.009 \\
\hline Model 5 & 1 & $1.29(0.56-3.00)$ & $1.69(0.77-3.73)$ & $2.42(1.15-5.10)^{\S}$ & 0.01 \\
\hline$\% \mathrm{PFEV}_{1}, n$ & 412 & 415 & 414 & 412 & \\
\hline Model 1 & 1 & $0.67(0.31-1.47)$ & $1.06(0.53-2.13)$ & $1.87(1.002-3.51)^{\S}$ & 0.02 \\
\hline Model 2 & 1 & $0.78(0.36-1.72)$ & $1.35(0.66-2.77)$ & $2.42(1.23-4.75)^{\S}$ & 0.003 \\
\hline Model 3 & 1 & $0.76(0.34-1.69)$ & $1.16(0.56-2.42)$ & $2.09(1.04-4.21)^{\S}$ & 0.02 \\
\hline Model 4 & 1 & $0.75(0.34-1.69)$ & $1.10(0.53-2.31)$ & $2.00(0.99-4.0 .4)$ & 0.02 \\
\hline Model 5 & 1 & $0.76(0.34-1.70)$ & $1.13(0.54-2.35)$ & $2.00(0.99-4.06)$ & 0.02 \\
\hline $\mathrm{FEV}_{1} / \mathrm{FVC}, n$ & 412 & 413 & 416 & 412 & \\
\hline Model 1 & 1 & $1.41(0.76-2.63)$ & $0.71(0.34-1.46)$ & $0.94(0.48-1.86)$ & 0.42 \\
\hline Model 2 & 1 & $1.38(0.74-2.59)$ & $0.60(0.28-1.26)$ & $0.74(0.36-1.56)$ & 0.15 \\
\hline Model 3 & 1 & $1.56(0.82-2.98)$ & $0.69(0.32-1.48)$ & $0.94(0.44-1.98)$ & 0.41 \\
\hline Model 4 & 1 & $1.52(0.80-2.91)$ & $0.69(0.32-1.49)$ & $0.94(0.44-1.98)$ & 0.42 \\
\hline Model 5 & 1 & $1.55(0.81-2.97)$ & $0.69(0.32-1.50)$ & $0.93(0.44-1.97)$ & 0.41 \\
\hline
\end{tabular}

Data are expressed as odds ratio (95\% confidence interval) with references of highest quartiles (Q1).

Model 1: unadjusted; Model 2: adjusted for age, sex, height, and current smoking (versus nonsmokers); Model 3: Model 2 plus adjustment for daily alcohol consumption (versus infrequent/no alcohol consumption), regular exercise (versus no regular exercise), waist circumference, log-transformed CRP, and selfreported past history of stroke; Model 4: Model 3 plus adjustment for diabetes; Model 5: Model 3 plus adjustment for MetS.

* $P$ values correspond to tests for linear trends across quartile treated as a continuous value.

${ }^{\dagger} P<0.005,{ }^{\S} P<0.05$ for each association.

\section{Discussion}

This study was conducted to investigate the relationship of impaired lung functions with resting electrocardiographic ST-T abnormalities in a cross-sectional analysis of asymptomatic adults without past history of heart disease. We found that LFVC and probably low $\mathrm{FEV}_{1}$, but not low $\mathrm{FEV}_{1} / \mathrm{FVC}$, were significantly associated with ST-T abnormalities, which was attenuated by further adjustment for cardiometabolic risk factors, diabetes (presumably mostly type 2 diabetes in this study), or MetS, but remained significant. Notably, cardiometabolic risk factors included circulating CRP and waist circumference, surrogate markers of systemic inflammation, and amount of abdominal fat, respectively. Collectively, the current findings suggest that LFVC, which often reflects restrictive pulmonary function pattern, may be independently associated with ST-T abnormalities irrespective of clinical confounders, diabetes, and MetS, whereas obstructive pulmonary function defects are not.

So far, no clinical study has examined the relationship of LFVC with electrocardiographic abnormalities with the exception of a study by Sideris and Katsadoros [23]. This study reported a negative correlation between vital capacity and number of abnormalities, such as a rightward shift of the P-wave and clockwise rotation of QRS, but it did not address ST or T-wave abnormalities. Thus, to our knowledge, this study is the first to demonstrate the association between LFVC and ST-T abnormalities, which is conceivably prognostic for cardiovascular events in asymptomatic people $[14,16,17]$.

According to previous studies, LFVC and restrictive pulmonary defects have been associated with coronary artery calcification [5] and arterial stiffness [3, 4]. Because artery calcification and arterial stiffness generally reflect atherosclerosis and ischemic cardiovascular diseases, the current results are consistent with these previous studies. Generally, ST-T abnormalities are observed in various conditions, such as ischemia, hypokalemia, cardiomyopathy, and pulmonary embolism [15]. Ohira et al. [13] mentioned that minor ST-T abnormalities may reflect an end-organ effect of long-term hypertension because hypertensive men with minor ST-T abnormalities tended to have longer durations of hypertension in their study. Indeed, arterial stiffness assessed with pulse-wave velocity is substantially affected by hypertension [24]. Furthermore, a recent study showed that decline in FVC predicted incident hypertension in young apparently healthy individuals [22]. Actually, in our study, LFVC was robustly associated with hypertension, even after full adjustments for critical confounders. Nevertheless, the significant association between LFVC and ST-T abnormalities persisted after further adjustments for confounders plus hypertension or medication for hypertension. Therefore, although hypertension likely contributes in part to the observed associations through the close interrelationship of lung with cardiovascular system, other unknown factors may 
principally interfere with the associations between LFVC and ST-T abnormalities.

Considering that LFVC was associated with ST-T abnormalities independently of cardiometabolic factors, circulating CRP, diabetes, and MetS, which are proatherosclerotic and proinflammatory $[9,10]$, several factors not examined in this study, such as insulin resistance, oxidative stress, or subclinical hypoxia, might interfere with the relationship between LFVC and ST-T abnormalities. In accordance with this, previous studies have hypothesized that insulin resistance is a fundamental element for the pathophysiology of LFVC and restrictive lung function $[2,6,7]$.

Meanwhile, many studies in the past decade have shown that a predisposition for cardiovascular disease and impaired pulmonary functions (low vital capacity and low $\mathrm{FEV}_{1}$ ) are associated with low birth weight $[25,26]$, possibly via physiological alterations such as increased adrenocortical and sympathoadrenal responses to an adverse fetal environment [27]. LFVC and ST-T abnormalities might then relate to such potential factors as epiphenomena.

Alternatively, physicochemical factors might interfere with the associations. Of note, the American Heart Association recently updated its scientific statement to describe that exposure to particulate-matter air pollution contributes to cardiovascular morbidity and mortality [28]. It highlighted several possible biological mechanisms secondary to pulmonary oxidative stress, inflammation, and an impaired lung autonomic nervous system that might result in vascular dysfunction and ST-segment depression irrespective of metabolic abnormalities.

Several limitations should be mentioned. First, because of the nature of cross-sectional studies, causality remains unknown and must be elucidated in large prospective studies. Second, it was not possible to distinguish between major and minor ST-T abnormalities in this study because the electrocardiogram findings on individual checkup sheets in combinations of major and minor ST-T abnormalities were recorded together indistinguishably. However, most ST$\mathrm{T}$ abnormalities were likely to be minor ST-T abnormalities because the prevalence of minor ST-T abnormalities is approximately 2-fold greater than that of major ST-T abnormalities in the Japanese population [13].

Third, all subjects in this study were instructed by staff members trained in spirometry, and the subjects performed a few rehearsals. Nevertheless, it was not clear whether all subjects had acceptable results in the actual test, especially the elderly, regardless of cognitive status, physical performance, or education level. This is likely to result in potential bias of the outcomes.

Finally, most subjects were healthy with good profiles in terms of various parameters, although a small portion of them had several critical conditions such as diabetes or history of stroke. Therefore, the current findings may not be applicable to other populations that have more cardiometabolic risk factors and ST-T abnormalities, where the observed associations, if any, might be dependent on hypertension, diabetes, or metabolic syndrome.

\section{Conclusion}

LFVC may be associated with ST-T abnormalities independently of cardiometabolic risk factors, diabetes, and MetS in asymptomatic adults without overt heart disease. Our results suggest that there is a mechanism linking low vital capacity with common electrocardiographic abnormalities and that this mechanism is prognostic for increased risk for cardiovascular diseases, which needs to be confirmed in further larger studies.

\section{Disclosure}

The present research was not supported by specific grants from any funding agency in the public, commercial, or notfor-profit sectors.

\section{Conflict of Interests}

The authors declare no conflict of interests.

\section{References}

[1] S. Guerra, D. L. Sherrill, C. Venker, C. M. Ceccato, M. Halonen, and F. D. Martinez, "Morbidity and mortality associated with the restrictive spirometric pattern: a longitudinal study," Thorax, vol. 65, no. 6, pp. 499-504, 2010.

[2] F. L. Fimognari, S. Scarlata, and R. Antonelli-Incalzi, "Why are people with "poor lung function" at increased atherothrombotic risk? A critical review with potential therapeutic indications," Current Vascular Pharmacology, vol. 8, no. 4, pp. 573586, 2010.

[3] M. Zureik, A. Benetos, C. Neukirch et al., "Reduced pulmonary function is associated with central arterial stiffness in men," American Journal of Respiratory and Critical Care Medicine, vol. 164, no. 12, pp. 2181-2185, 2002.

[4] C. E. Bolton, J. R. Cockcroft, R. Sabit et al., "Lung function in mid-life compared with later life is a stronger predictor of arterial stiffness in men: the Caerphilly Prospective Study," International Journal of Epidemiology, vol. 38, no. 3, pp. 867876, 2009.

[5] H. Y. Park, S. Y. Lim, J. H. Hwang et al., "Lung function, coronary artery calcification, and metabolic syndrome in 4905 Korean males," Respiratory Medicine, vol. 104, no. 9, pp. 13261335, 2010.

[6] D. A. Lawlor, S. Ebrahim, and G. D. Smith, "Associations of measures of lung function with insulin resistance and Type 2 diabetes: findings from the British Women's Heart and Health Study," Diabetologia, vol. 47, no. 2, pp. 195-203, 2004.

[7] F. L. Fimognari, P. Pasqualetti, L. Moro et al., "The association between metabolic syndrome and restrictive ventilatory dysfunction in older persons," Journals of Gerontology A, vol. 62, no. 7, pp. 760-765, 2007.

[8] K. Nakajima, Y. Kubouchi, T. Muneyuki, M. Ebata, S. Eguchi, and H. Munakata, "A possible association between suspected restrictive pattern as assessed by ordinary pulmonary function test and the metabolic syndrome," Chest, vol. 134, no. 4, pp. 712-718, 2008.

[9] S. M. Grundy, J. I. Cleeman, S. R. Daniels et al., "Diagnosis and management of the metabolic syndrome: an American Heart Association/National Heart, Lung, and Blood Institute 
scientific statement," Circulation, vol. 112, no. 17, pp. 27352752, 2005.

[10] S. Mottillo, K. B. Filion, J. Genest et al., "The metabolic syndrome and cardiovascular risk: a systematic review and meta-analysis," Journal of the American College of Cardiology, vol. 56, no. 14, pp. 1113-1132, 2010.

[11] K. Cullen, N. S. Stenhouse, K. L. Wearne, and G. N. Cumpston, "Electrocardiograms and 13 year cardiovascular mortality in Busselton study," British Heart Journal, vol. 47, no. 3, pp. 209212, 1982.

[12] Y. Liao, K. Liu, A. Dyer et al., "Sex differential in the relationship of electrocardiographic ST-T abnormalities to risk of coronary death: 11.5 year follow-up findings of the Chicago Heart Association Detection Project in Industry," Circulation, vol. 75, pp. 347-352, 1987.

[13] T. Ohira, H. Iso, H. Imano et al., "Prospective study of major and minor ST-T abnormalities and risk of stroke among Japanese," Stroke, vol. 34, no. 12, pp. e250-e253, 2003.

[14] A. Kumar and D. M. Lloyd-Jones, "Clinical significance of minor nonspecific ST-segment and T-wave abnormalities in asymptomatic subjects: a systematic review," Cardiology in Review, vol. 15, no. 3, pp. 133-142, 2007.

[15] E. B. Hanna and D. L. Glancy, "ST-segment depression and T-wave inversion: classification, differential diagnosis, and caveats," Cleveland Clinic Journal of Medicine, vol. 78, no. 6, pp. 404-414, 2011.

[16] M. L. Daviglus, Y. Liao, P. Greenland et al., "Association of nonspecific minor ST-T abnormalities with cardiovascular mortality: the Chicago western electric study," Journal of the American Medical Association, vol. 281, no. 6, pp. 530-536, 1999.

[17] P. Greenland, X. Xie, K. Liu et al., "Impact of minor electrocardiographic ST-segment and/or T-wave abnormalities on cardiovascular mortality during long-term follow-up," American Journal of Cardiology, vol. 91, no. 9, pp. 1068-1074, 2003.

[18] American Diabetes Association, "Diagnosis and classification of diabetes mellitus," Diabetes Care, vol. 33, supplement 1, pp. S62-S69, 2010.

[19] Y. Seino, K. Nanjo, N. Tajima et al., "Report of the committee on the classification and diagnostic criteria of diabetes mellitus," Journal of Diabetes Investigation, vol. 1, no. 5, pp. 212228, 2010.

[20] Japanese Respiratory Society, Reference values of spirogram and arterial blood gas levels in Japanese, Japanese Respiratory Society, Tokyo, Japan, 2001.

[21] R. J. Prineas, R. S. Crow, and H. Blackburn, The Minnesota Code Manual of Electrocardiographic Findings: Standards and Procedures for Measurement and Classification, John WrightPSG, Littleton, Mass, USA, 1982.

[22] D. R. Jacobs Jr., H. Yatsuya, M. O. Hearst et al., "Rate of decline of forced vital capacity predicts future arterial hypertension: the coronary artery risk development in young adults study," Hypertension, vol. 59, pp. 219-225, 2012.

[23] D. A. Sideris and D. P. Katsadoros, "Some correlations between electrocardiographic findings and lung volumes in pulmonary diseases," Journal of Electrocardiology, vol. 7, no. 4, pp. 295300, 1974.

[24] A. Milan, F. Tosello, A. Fabbri et al., "Arterial stiffness: from physiology to clinical implications," High Blood Pressure and Cardiovascular Prevention, vol. 18, no. 1, pp. 1-12, 2011.

[25] R. J. Hancox, R. Poulton, J. M. Greene, C. R. McLachlan, M. S. Pearce, and M. R. Sears, "Associations between birth weight, early childhood weight gain and adult lung function," Thorax, vol. 64, no. 3, pp. 228-232, 2009.

[26] L. Pei, G. Chen, J. Mi et al., "Low birth weight and lung function in adulthood: retrospective cohort study in China, 1948-1996," Pediatrics, vol. 125, no. 4, pp. e899-e905, 2010.

[27] D. I. W. Phillips, A. Jones, and P. A. Goulden, "Birth weight, stress, and the metabolic syndrome in adult life," Annals of the New York Academy of Sciences, vol. 1083, pp. 28-36, 2006.

[28] R. D. Brook, S. Rajagopalan, C. A. Pope III et al., "Particulate matter air pollution and cardiovascular disease: an update to the scientific statement from the American Heart Association," Circulation, vol. 121, pp. 2331-2378, 2010. 


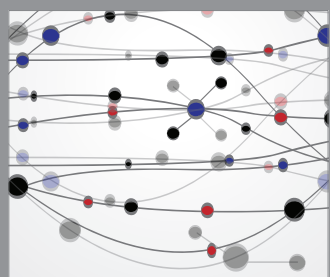

The Scientific World Journal
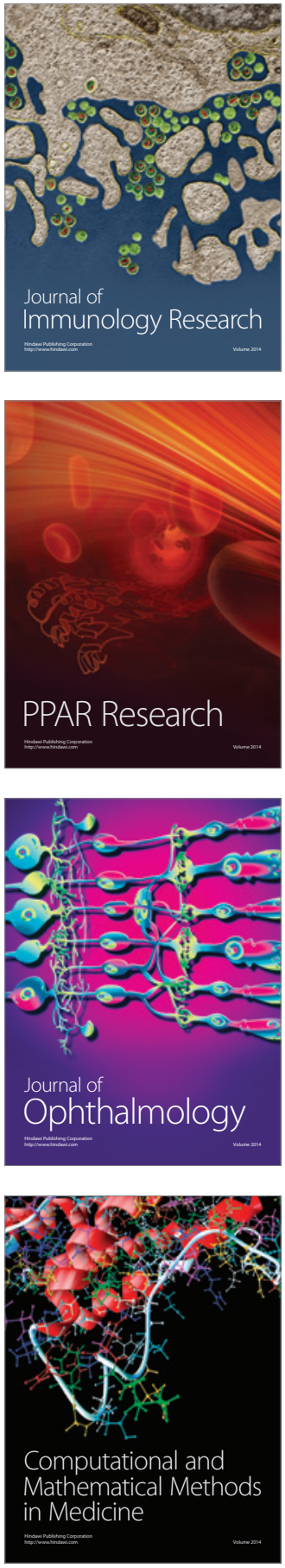

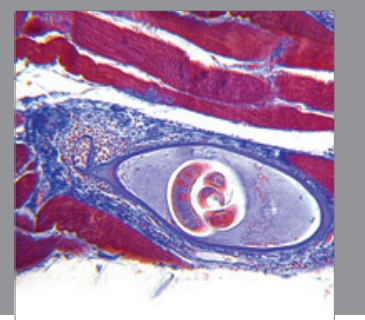

Gastroenterology

Research and Practice
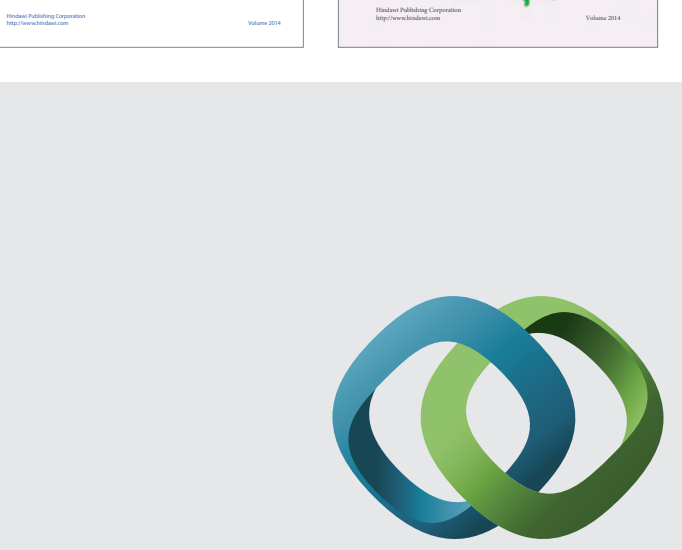

\section{Hindawi}

Submit your manuscripts at

http://www.hindawi.com
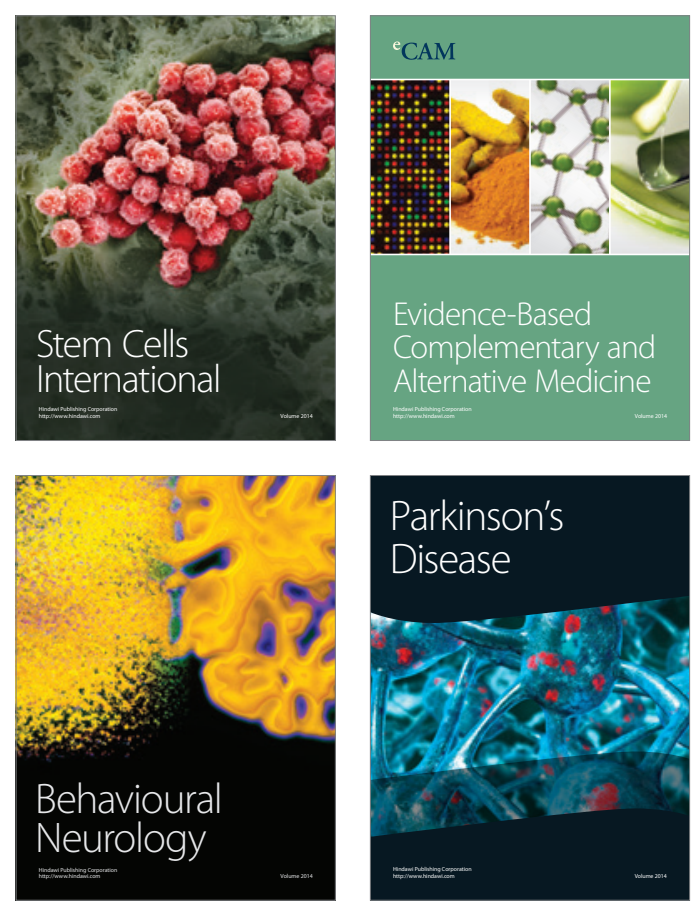

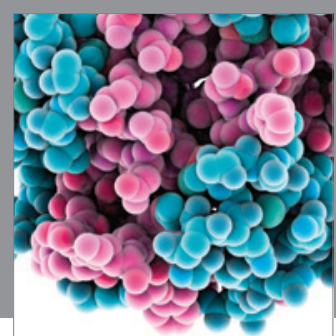

Journal of
Diabetes Research

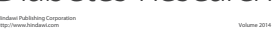

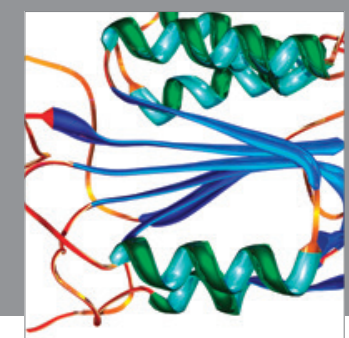

Disease Markers
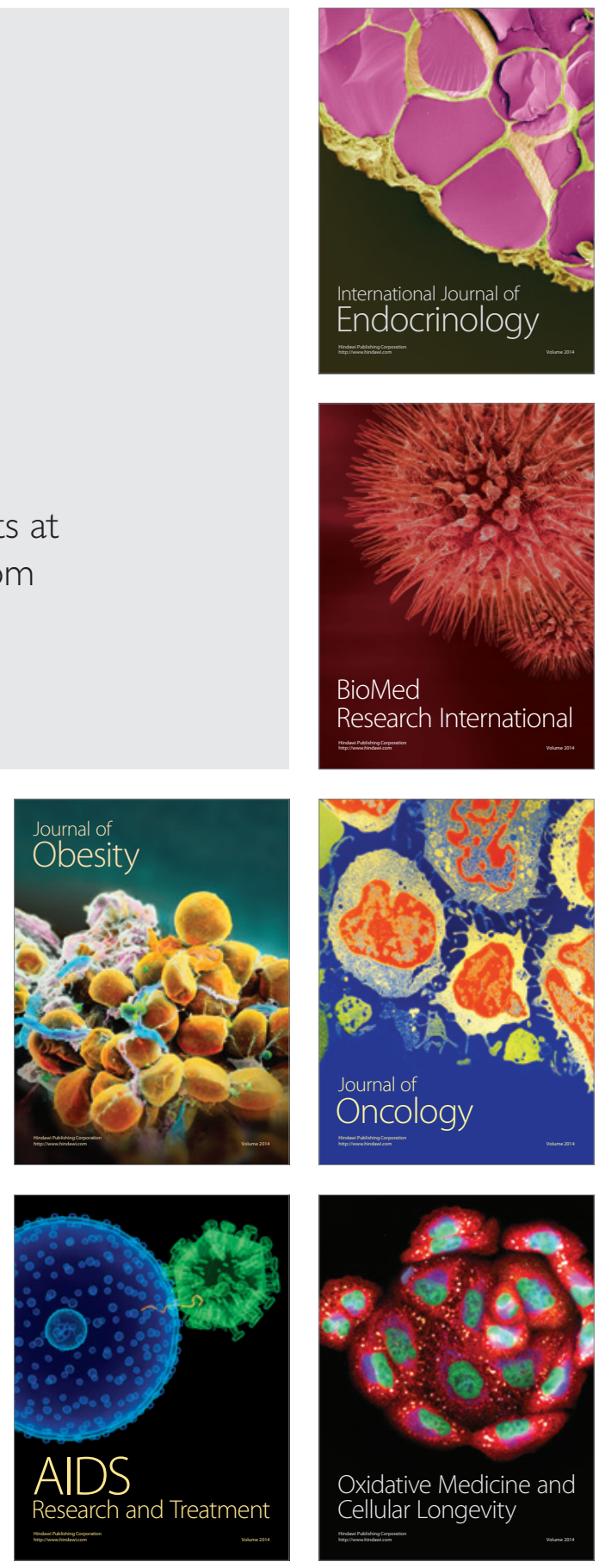